\title{
Philosophiques
}

\section{Rationalité et affectivité des intuitions}

\section{ANNE MEYLAN}

Volume 44, numéro 1, printemps 2017

Nature, rôle et importance des intuitions

URI : https://id.erudit.org/iderudit/1040326ar

DOI : https://doi.org/10.7202/1040326ar

Aller au sommaire du numéro

Éditeur(s)

Société de philosophie du Québec

ISSN

0316-2923 (imprimé)

1492-1391 (numérique)

Découvrir la revue

Citer cet article

Meylan, A. (2017). Rationalité et affectivité des intuitions. Philosophiques, 44(1), 31-47. https://doi.org/10.7202/1040326ar

\section{Résumé de l'article}

Cette contribution a deux objectifs principaux. Le premier est de montrer que les intuitions sont caractérisées par ce que j’appellerai " une capacité rationnelle », c'est-à-dire, qu'elles sont susceptibles d'être évaluées quant à leur rationalité ou leur irrationalité. Le second objectif de cet article est d'étayer l'hypothèse selon laquelle les intuitions seraient des états affectifs proches des émotions - et non pas des états doxastiques ou des expériences perceptuelles -, en montrant qu'une telle conception affective des intuitions est seule capable de rendre compte de (i) la spécificité phénoménologique, (ii) la modularité, et (iii) la capacité rationnelle des intuitions.
Ce document est protégé par la loi sur le droit d'auteur. L'utilisation des services d'Érudit (y compris la reproduction) est assujettie à sa politique d'utilisation que vous pouvez consulter en ligne.

https://apropos.erudit.org/fr/usagers/politique-dutilisation/ 


\title{
Rationalité et affectivité des intuitions
}

\author{
ANNE MEYLAN \\ Université de Bâle
}

\begin{abstract}
RÉSUMÉ. - Cette contribution a deux objectifs principaux. Le premier est de montrer que les intuitions sont caractérisées par ce que j'appellerai «une capacité rationnelle», c'est-à-dire, qu'elles sont susceptibles d'être évaluées quant à leur rationalité ou leur irrationalité. Le second objectif de cet article est d'étayer l'hypothèse selon laquelle les intuitions seraient des états affectifs proches des émotions - et non pas des états doxastiques ou des expériences perceptuelles -, en montrant qu'une telle conception affective des intuitions est seule capable de rendre compte de (i) la spécificité phénoménologique, (ii) la modularité, et (iii) la capacité rationnelle des intuitions.
\end{abstract}

\begin{abstract}
This article has two purposes. First, it wants to show that intuitions possess, what I shall call, "a rational capacity" : they are mental states susceptible to be assessed as rational or irrational. Second, this contribution aims at providing some evidence supporting the view that intuitions are affective states similar to emotions rather than doxastic or perceptual states. Such an affective account of the intuitions is the only one able to capture (i) the specific phenomenology, (ii) the modularity and (iii) the rational capacity of the intuitions.'
\end{abstract}

\section{Introduction}

Contrairement à la capacité des intuitions à justifier les croyances, et à leur fiabilité, la capacité des intuitions à être elles-mêmes évaluées quant à leur ir/ rationalité n'a, à notre connaissance, peu ou pas vraiment été discutée. Le premier objectif de cette contribution est de montrer que les intuitions ont une telle capacité rationnelle, c'est-à-dire qu'elles sont - à l'instar, par exemple, des croyances — susceptibles d'être évaluées quant à leur ir/rationalité ${ }^{2}$ La raison de penser que les intuitions ont une capacité rationnelle est présentée dans le $\mathbb{S} 2$ ci-dessous. Avant cela, nous croyons utile de procéder à trois remarques préliminaires.

Tout d'abord, il faut prendre garde de distinguer: I) «la capacité rationnelle d'un type d'état mental », de 2) «la rationalité de tel ou tel token tombant sous le type en question». La seconde expression ne désigne pas

1. Merci à Julien Deonna, Olivier Massin, Kevin Mulligan, Christine Tappolet, Fabrice Teroni pour leur aide sur l'une ou l'autre des affirmations contenues dans cet article. Il va de soi que la responsabilité des erreurs possibles revient, par contre, entièrement à l'auteur.

2. Nous employons les expressions «capacité rationnelle" et "rationalité » là où d'autres emploieraient peut-être les expressions «capacité justificatrice» et «justification». Nous préférons utiliser le terme de rationalité car, comme nous l'expliquons ci-dessous, la question de la capacité rationnelle des intuitions dont nous traitons dans cette contribution est distincte de la question, plus discutée, de leur fiabilité. Or la justification est parfois identifiée à la fiabilité (par exemple dans Goldman I979). 
une capacité mais le fait actuel, pour tel ou tel token d'état mental, d'être rationnel, c'est-à-dire d'être entretenu pour une ou plusieurs raisons.

\section{Capacité rationnelle}

Un type d'état mental $E$ a une capacité rationnelle si et seulement si $E$ est un type d'état mental dans lequel il est possible d'être pour certaines raisons.

\section{Rationalité}

L'état mental $e$ que possède un sujet $S$ au temps $t$ est rationnel si et seulement si $S$ est dans $e$ pour certaines raisons.

\section{Irrationalité}

L'état mental $e$ que possède un sujet $S$ au temps $t$ est irrationnel si et seulement si (i) le type d'état mental $E$ sous lequel $e$ tombe a une capacité rationnelle, et (ii) $S$ est dans $e$ sans aucune raison ${ }^{3}$.

La rationalité ou l'irrationalité d'un état mental $e$ (token) présuppose la capacité rationnelle du type d'état mental $E$ sous lequel $e$ tombe.

La deuxième remarque préliminaire est la suivante. Il est important de distinguer la question de la capacité rationnelle des intuitions de la question, beaucoup plus souvent discutée, qui porte sur leur capacité justifiante ou rationalisante, c'est-à-dire, de la question suivante: les intuitions sont-elles susceptibles de justifier ou de rationaliser d'autres croyances? Le fait qu'un type d'état mental ait une capacité justificatrice ou rationalisante n'implique pas qu'il ait une capacité rationnelle. Il est possible qu'un type d'état mental ait la capacité de justifier d'autres croyances sans qu'il soit lui-même susceptible d'être évalué quant à sa rationalité. Par exemple, de nos jours, les philosophes pensent couramment que les expériences perceptuelles sont, dans des circonstances ordinaires, en mesure de justifier les croyances qui en résultent. Par exemple, mon expérience visuelle que ce costume est vert sapin est susceptible de justifier la croyance qu'il est vert sapin. Pourtant, comme nous le verrons plus en détail ci-dessous, les expériences perceptuelles ne sont pas susceptibles d'être évaluées sur le plan de leur rationalité. $\mathrm{Si}$, comme l'illustre le cas des expériences perceptuelles, la capacité rationalisante ou justificatrice d'un type d'état mental n'implique pas que celui-ci soit susceptible d'être évalué quant à sa rationalité, il n'est évidemment pas possible de nous appuyer sur les arguments qui cherchent à établir la capa-

3. Une autre manière (plus sévère) de formuler la norme de rationalité consisterait à affirmer que celle-ci requiert non seulement des raisons mais de bonnes raisons. Une autre manière (moins sévère) de formuler la norme d'irrationalité consisterait à affirmer que celle-ci requiert de mauvaises raisons. Puisque rien de ce que nous affirmons ci-dessous ne dépend de la manière précise dont nous formulons les normes de rationalité et d'irrationalité, le lecteur est libre d'opter pour celles qu'il ou elle préfère. 
cité rationalisante des intuitions pour prouver que ces dernières ont une capacité rationnelle. Il nous faut trouver une autre raison. C'est la tâche à laquelle nous nous attelons dans la section $\mathbb{2}$.

Finalement, il est important de souligner que la question qui nous intéresse - celle qui porte sur la capacité rationnelle des intuitions - est complètement indépendante du problème, beaucoup plus discuté, qui concerne leur fiabilité. Cela constitue notre troisième et dernière remarque préliminaire. La fiabilité des intuitions, principalement celle des intuitions morales, a et fait toujours l'objet d'un large débat philosophique. Cela s'explique aisément. Si vous supposez que la croyance vraie que $p$ est une connaissance seulement si la croyance vraie que $p$ résulte d'un processus ou d'une compétence fiable - c'est-à-dire d'un processus ou d'une compétence conduisant le plus souvent à l'acquisition de croyances vraies - la preuve de la fiabilité des intuitions doit être faite pour qu'elles puissent être légitimement considérées comme sources de connaissance. Mais — et c'est là l'élément important - ce que nous affirmons ci-dessous en ce qui concerne la capacité rationnelle des intuitions n'a d'influence ni sur la question de la légitimité des intuitions en tant que sources de connaissance ni sur la question, connexe, de leur fiabilité. Les normes de rationalité et d'irrationalité présentées ci-dessus sont des normes d'évaluation des états mentaux parfaitement compatibles avec l'hypothèse (externaliste) selon laquelle la rationalité des croyances ne joue aucun rôle dans l'acquisition de connaissances ${ }^{4}$. S'interroger sur la capacité rationnelle des intuitions revient simplement à se demander si les intuitions sont susceptibles d'être évaluées par ces normes, c'est-à-dire, si les intuitions sont des états mentaux dans lesquels il est possible d'être pour certaines raisons. Et cela, clairement, ne nous dit rien de la légitimité ou de l'illégitimité des intuitions comme sources de connaissance. Par ailleurs, la réponse affirmative que nous nous apprêtons à donner à la question: "Les intuitions sont-elles susceptibles d'être évaluées quant à leur ir/rationalité ?» ne fournit aucune raison de penser qu'elles sont, ou ne sont pas, fiables. Il est, en effet, des états mentaux fiables qui ne sont pas susceptibles d'être évalués quant à leur rationalité. Mon expérience visuelle que ce costume est vert sapin, dans des circonstances normales, est l'un d'eux. À l'inverse, certains états mentaux qui ne sont pas fiables peuvent néanmoins être qualifiés de rationnels ou d'irrationnels. Si je suis, à mon insu, un cerveau dans une cuve, la croyance que ce costume est vert sapin - entretenue sur la base de ce que je considère (faussement) comme une expérience visuelle de vert sapin - est un état mental rationnel mais non fiable.

4. Cela n'enlève rien à la portée des normes de rationalité et d'irrationalité. Entretenir des croyances rationnelles et/ou éviter d'entretenir des croyances irrationnelles reste un objectif important, même dans l'hypothèse externaliste. 


\section{La capacité rationnelle des intuitions}

La raison principale de penser que, contrairement aux expériences perceptuelles, les intuitions sont des états mentaux dont la rationalité ou l'irrationalité est susceptible d'être évaluée, est qu'il semble possible - même si cela n'a, à ma connaissance, jamais été clairement souligné - de poser la question «pourquoi? » ou "pour quelle(s) raison(s)?» à leur propos. Le fait qu'il soit possible de poser la question du pourquoi dans le cas des intuitions atteste de leur capacité à être évaluées quant à leur ir/rationalité. C'est ce que nous baptiserons ci-dessous «le critère du pourquoi » ${ }^{5}$.

Avant d'envisager les intuitions, commençons par appliquer ce même critère aux expériences perceptuelles. Supposons que Julie cherche à déterminer quelles sont les raisons pour lesquelles Paul croit que le costume qui se trouve devant lui est vert sapin, qu'elle lui demande, à cette fin: "Pourquoi crois-tu que ce costume est vert sapin? ? et que Paul réponde: "Parce que je vois qu'il est vert sapin". Cette réponse met un terme à l'investigation de Julie. Il est, en effet, impossible de prolonger le dialogue en demandant à Paul pourquoi/pour quelle raison il fait cette expérience perceptuelle ou pourquoi/pour quelle raison il voit du vert sapin. Ces questions ne font pas sens, car les expériences perceptuelles ne sont pas des états mentaux qu'il est possible de posséder pour des raisons, c'est-à-dire de manière rationnelle ou irrationnelle. Autrement dit, la raison pour laquelle ces questions ne font pas sens est que les expériences perceptuelles sont, comme nous l'avons d'ores et déjà mentionné, dénuées de capacité rationnelle. La non-satisfaction du critère du pourquoi est une marque de l'incapacité des expériences perceptuelles à être évaluées sur le plan de leur ir/rationalité.

La conclusion est-elle la même en ce qui concerne les intuitions? Nous ne le pensons pas. Et nous souhaitons en faire la preuve en nous appuyant sur deux exemples distincts.

Imaginons que Jules soit apprenti philosophe et qu'il teste les intuitions de sa grand-mère en lui présentant le célèbre cas des fausses granges de Goldman ${ }^{6}$. À la suite de cette présentation, sa grand-mère affirme que l'individu qui s'arrête devant la seule vraie grange alors qu'il se promène dans un environnement composé de milliers de fausses granges (sauf une) ne sait pas qu'il s'agit là d'une vraie grange. Supposons maintenant que Jules lui demande pourquoi elle pense que l'individu en question ne sait pas cela et que sa grand-mère lui réponde: "J'ai l'intuition que c'est le cas.» Cette réponse clôt-elle le questionnement de Jules de la même manière que la réponse "parce que je vois que $\mathrm{X}$ est vert sapin» met, en effet, un terme définitif à l'investigation des raisons pour lesquelles un individu croit que $\mathrm{X}$

5. Deonna et Teroni (20I2a, chap. VIII) font usage du critère du pourquoi pour faire la preuve du fait que les émotions peuvent être évaluées comme justifiées ou injustifiées. Voir également Tappolet 2016, 40-I.

6. Le cas est présenté dans Goldman 1976. 
est vert sapin. Il nous semble que non. La question: "Pourquoi as-tu l'intuition que l'individu des fausses granges ne sait pas?" fait sens. Il est tout à fait envisageable que la grand-mère de Jules fournisse des réponses à cette question. Nous pourrions, par exemple, imaginer qu'elle donne la réponse suivante: «Parce que j'ai imaginé cet individu au milieu de toutes ces fausses granges.»

Notre second exemple est tiré d'un article récent d'Eliah Chudnoff. Chudnoff présente un cas dans lequel un individu prénommé Albert a l'intuition de la vérité géométrique suivante: chaque diamètre d'un cercle constitue l'une de ses lignes de symétrie. Voici — et c'est un point important — comment, d'après Chudnoff, Albert acquiert cette intuition.

Albert considers the proposition: 'every diameter of a circle determines a line of symmetry for it'. At first, he does not know what to think about it. To tell whether the proposition is true, [...] Albert exercises his visual imagination. He imagines an arbitrary circle and some of its diameters. Circles are shapes of this kind, and diameters are line segments like these that span the circle and pass through its midpoint [...] As Albert thinks and imagines his way toward a better awareness of what circles are, and what lines of symmetry are [...] his thoughts and imaginings click together in a way that makes it intuitively appear to him that every diameter of a circle does indeed determine a line of symmetry for it (Chudnoff, 20I I, 636-7).

Supposons maintenant que l'épouse d'Albert entre dans la pièce au moment précis auquel ce dernier s'exclame: "Mais bien sûr! Chaque diamètre d'un cercle constitue l'une de ses lignes de symétrie », qu'elle demande à Albert pourquoi il pense cela et que ce dernier lui réponde: "Parce que j'ai l'intuition que c'est le cas.» Une fois de plus, cette réponse ne semble pas mettre un terme au questionnement de sa femme de la même manière que la réponse "parce que je perçois que $p$ " met un terme définitif à l'investigation des raisons pour lesquelles un individu croit que $p$. Il paraît en effet possible de demander à Alfred pour quelle raison il a cette intuition. Et la raison qu'Albert mentionnera dans sa réponse à cette question lui est attribuée par Chudnoff dans la citation ci-dessus. La principale raison pour laquelle Albert a l'intuition que chaque diamètre d'un cercle constitue l'une de ses lignes de symétrie est qu'il a imaginé un cercle, ses diamètres et ses lignes de symétrie (en imaginant, par exemple, qu'il plie le cercle). Ce que ces deux exemples montrent est que, contrairement aux expériences perceptuelles, les intuitions satisfont le critère du pourquoi. Or le fait que les intuitions satisfont le critère du pourquoi est la preuve de leur capacité rationnelle, la preuve de leur capacité à être évaluées quant à leur ir/rationalité.

À cet instant, le lecteur pourrait douter que ce que la grand-mère de Jules et Albert mentionne dans ses réponses soit vraiment capable de rationaliser ses intuitions au même titre qu'une expérience perceptuelle ou que le témoignage d'autrui, par exemple, est capable de rationaliser une croyance. Il pourrait, en particulier, penser que ce que nous considérons comme des 
raisons qui rationalisent les intuitions de la grand-mère de Jules et d'Albert sont seulement des raisons qui expliquent causalement ces intuitions. Nous tâchons de résoudre cette difficulté à la fin de cette contribution $(\mathbb{S} 4.2)$. Avant de clore cette section, nous aimerions encore insister sur le fait que rien de tout ce qui vient d'être dit n'exclut que nous expérimentions ou entretenions - occasionnellement ou même souvent — certaines intuitions sans raison. Il s'agira alors, si nous appliquons le critère d'irrationalité mentionné dans le $\mathbb{I}$, d'intuitions irrationnelles. Mais il n'y aurait évidemment pas d'intuitions irrationnelles si les intuitions n'étaient pas caractérisées par une capacité rationnelle. Et c'est tout ce dont nous souhaitons faire la preuve ici. Les intuitions semblent, contrairement aux expériences perceptuelles, pouvoir être évaluées quant à leur ir/rationalité.

\section{Conception doxastique et perceptive des intuitions}

Les intuitions sont des états mentaux. Mais de quel type d'états mentaux s'agit-il? Deux principales conceptions de la nature des intuitions s'affrontent aujourd'hui. Selon la première d'entre elles — baptisée parfois la conception doxastique des intuitions - les intuitions sont des états doxastiques: soit des croyances, soit des dispositions à croire ${ }^{7}$.

\section{Conception doxastique des intuitions}

$S$ a l'intuition que $p=D f, S$ croit ou est disposé à croire que $p$.

Selon une autre conception - que j'appellerai «conception perceptive» des intuitions -, il existe ce que nous appellerons des expériences intuitives qui constituent - au même titre, par exemple, que les expériences visuelles - un type d'expériences perceptuelles ${ }^{8}$.

\section{Conception perceptive des intuitions}

$S$ a l'intuition que $p=D f, S$ fait l'expérience intuitive que $p$.

Les tenants de la conception perceptive ont suggéré deux raisons principales de préférer cette dernière à la conception doxastique des intuitions. Notons bien que nous ne supposons pas que les raisons en question soient concluantes. Réfuter la conception doxastique n'est pas l'objectif de cette section. Nous indiquons quelles sont les deux objections les plus fortes de la conception perceptualiste à l'encontre de la conception doxastique, dans le but plus modeste de mettre en lumière les desiderata les plus importants qu'une conception de la nature des intuitions doit apparemment satisfaire.

7. Des versions de la conception doxastique sont défendues, par exemple, dans Goldman and Pust I998, Williamson 2007.

8. Bealer (I996, I998), Chudnoff (20II, 20I3), et Huemer (2008) sont des défenseurs de la conception perceptive des intuitions. 
Selon Bealer (I996), mais également selon Chudnoff (20II), une des raisons principales de préférer la conception perceptive à la conception doxastique des intuitions est d'ordre phénoménologique. Les intuitions sont caractérisées par une phénoménologie différente de celle des croyances. Quelle est cette phénoménologie spécifique? Voici ce qu'en dit Chudnoff: "[It] is one in which it both seems to you that $p$ and in which you seem to be [...] aware of an item that makes it true that $p$ "(Chudnoff 20I I, 639).

Une intuition, par exemple l'intuition que les diamètres d'un cercle constituent ses lignes de symétrie (p), est un état mental dans lequel il nous semble non seulement que $p$ est vraie, mais il nous semble également être conscient de ce qui rend $p$ vraie. Lorsque nous croyons que $p$, il nous semble que $p$ est vraie, mais il ne nous semble pas forcément être conscients de ce qui rend $p$ vraie. Quand Clémence entend dire qu'il va faire beau demain $(q)$ et le croit, il lui semble que $q$ est vraie, mais il ne lui semble pas être consciente de ce qui rend $q$ vraie. Selon Chudnoff, la phénoménologie spécifique des intuitions caractérise bon nombre de nos autres expériences perceptuelles (mais pas toutes comme nous allons le voir d'ici peu). Lorsque Jules perçoit visuellement que le costume est vert sapin $(r)$, il lui semble que $r$ est vraie, et il lui semble également être conscient de ce qui rend $r$ vraie. Par contre, lorsque Jeanne perçoit qu'il va faire beau demain $(s)$ en regardant le baromètre, il lui semble que $s$ est vraie, mais il ne lui semble pas être consciente de ce qui rend $s$ vraie.

La seconde objection qu'il est possible de faire à la conception doxastique des intuitions est analogue à celle qui a été faite à l'encontre de la thèse selon laquelle les expériences visuelles, olfactives, etc., sont en fait des états doxastiques ${ }^{9}$. Lorsque nous percevons les fameuses lignes (ou flèches) de Müller-Lyer, nous faisons l'expérience de deux lignes de longueur différente mais, en général, nous ne croyons pas ni ne sommes disposés à croire que c'est le cas. Il est, dès lors, possible de faire l'expérience que $p$ sans croire ou être disposé à croire que $p$. Cela contredit directement la thèse selon laquelle les expériences visuelles, olfactives, etc., sont en fait des états doxastiques. La même chose est vraie des intuitions. Considérez par exemple ce que suggère Bealer (I996, 6), l'axiome (A):

(A) Pour toute condition $x$, il existe un ensemble contenant toutes les choses qui remplissent cette condition, et seulement elles.

Bealer sait que A est faux parce qu'il connaît les paradoxes de la théorie des ensembles. Comme il sait cela, il ne croit ni n'est disposé à croire que A. Pourtant, affirme Bealer, quand il considère A, il a l'intuition que A est vrai. Il est, dès lors, possible d'avoir l'intuition que $p$ sans croire ou être

9. Voir Bealer I996, 6, et Chudnoff 20I I, 63I-4 pour une objection de ce type, ainsi que Williamson 2007, 217, pour une réponse. 
disposé à croire que $p$. Nous baptiserons cette caractéristique des intuitions «la modularité des intuitions».

Le lecteur pourrait rétorquer qu'il y a moyen, pour la conception doxastique des intuitions, de répondre à cette seconde objection. Il pourrait également être en désaccord avec la description que fait Chudnoff de la phénoménologie des intuitions et/ou considérer que cela ne suffit pas à réfuter définitivement la conception doxastique. De nouveau, nous ne supposons pas ici que les deux objections mentionnées ci-dessus sont concluantes. Notre but est simplement de mettre en lumière quelques-uns des desiderata importants qu'une conception de la nature des intuitions doit, selon certains auteurs, satisfaire. Les voici donc. L'état mental en lequel consistent les intuitions doit:

I. être caractérisé par la phénoménologie spécifique mentionnée cidessus;

2. être modulaire;

À quoi, crucialement, il faut ajouter, si ce que nous avons affirmé dans le $\mathbb{S}$ 2 est correct:

3. avoir une capacité rationnelle.

Même si nous ne supposons pas qu'il est tout simplement impossible, pour la conception doxastique des intuitions, de satisfaire les desiderata I et 2, les objections ci-dessus prouvent que cela n'est du moins pas aisé. Quant à la conception perceptive des intuitions, elle est incapable de satisfaire le $3^{\text {e }}$ desideratum puisque, comme nous l'avons mentionné à plusieurs reprises, les expériences perceptuelles n'ont pas de capacité rationnelle. Le constat est donc le suivant: ni la conception doxastique ni la conception perceptive ne sortent totalement indemnes de l'examen auquel nous avons procédé cidessus. S'il existait une conception des intuitions capable de satisfaire les trois desiderata susmentionnés, elle bénéficierait, dès lors, d'un certain avantage théorique.

Nous pensons qu'il est possible d'élaborer une telle conception des intuitions. Il s'agit d'une conception qui les assimile à des états affectifs spécifiques, plus précisément à des états affectifs similaires à des émotions ${ }^{10}$. Avant de présenter cette hypothèse et d'essayer de l'étayer, il est important de rappeler que la nature des émotions a fait l'objet d'une controverse qui ressemble beaucoup à celle qui oppose la conception doxastique à la conception perceptive dans le cas des intuitions. Les émotions ont, elles aussi, été identifiées, parfois à des états doxastiques ${ }^{11}$, parfois à des expériences per-

10. Roeser (20II) se réclame d'une conception affective des intuitions.

11. Plus précisément à des jugements qui portent sur des propriétés évaluatives. Voir, par exemple, Nussbaum 200I, Solomon I980. 
ceptuelles ${ }^{12}$. Parfois, également, les émotions sont considérées comme des états mentaux sui generis. Il ne ferait évidemment pas sens de s'interroger sur la possibilité de rapprocher les intuitions des émotions, plutôt que des attitudes doxastiques ou des expériences perceptuelles, tout en supposant que les émotions sont des attitudes doxastiques ou des expériences perceptuelles. En ce sens, l'hypothèse selon laquelle les intuitions sont des états affectifs similaires à des émotions présuppose sans l'argumenter une conception non réductionniste des émotions selon laquelle les émotions sont des états mentaux sui generis ${ }^{13}$.

\section{Conception affective des intuitions}

\subsection{Les types d'états affectifs}

Les intuitions sont, selon la conception affective que nous souhaitons envisager ici, des états affectifs d'un type spécifique, des états affectifs similaires ${ }^{14}$ à des émotions.

Commençons dès lors par dire quelques mots sur ce qui distingue les émotions des autres types d'états affectifs. Il est courant de considérer, en plus des émotions, les humeurs et les sentiments comme appartenant à la catégorie des états affectifs. La différence cruciale entre les humeurs et les émotions est que, contrairement aux humeurs, les émotions sont des états intentionnels ${ }^{15}$. Les émotions portent sur des objets. On a peur du chien, on a honte de son action, on est jaloux de son voisin, on est surpris de sa gentillesse, etc. Qu'elle soit bonne, joviale, mauvaise, etc., notre humeur n'a pas d'objet. Les sentiments, quant à eux, peuvent être différenciés des émotions si l'on insiste sur leur aspect dispositionnel ${ }^{16}$. Les émotions ont lieu. Les émotions sont des épisodes affectifs occurrents alors que les sentiments sont des dispositions affectives dont la manifestation consiste en des épisodes affectifs occurrents, par exemple, mais pas exclusivement, en des émotions. L'amour, par exemple, est souvent considéré comme un sentiment. L'amour que l'épouse d'Albert éprouve à l'égard de son mari est une disposition dont les manifestations affectives possibles sont variées: admiration, pitié, jalousie, etc. La distinction entre les dispositions affectives - par exemple les sentiments - et les émotions est couramment omise. En effet, nous ne possédons souvent qu'un seul terme pour désigner la disposition affective et sa manifestation affective la plus évidente ${ }^{17}$. Lorsque j'affirme "Eugénie

12. Voir, par exemple, de Sousa I987; Tappolet 2000, 2016.

13. Selon la théorie attitudinale des émotions défendue par Deonna et Teroni (20I2a), par exemple, les émotions sont des états mentaux sui generis.

14. La raison pour laquelle nous parlons de similarité avec les émotions et non pas d'identité est que les intuitions ne possèdent pas certaines caractéristiques importantes que les émotions possèdent généralement. Nous expliquons cela au point $4 \cdot 3$.

15. Voir Deonna et Teroni 20I 2a, chap. I; Tappolet 20 I6.

16. Voir Deonna et Teroni 20 I2a, chap.I.

17. Ibid. 
admire Jeanne", je peux vouloir dire qu'Eugénie possède une disposition dont la manifestation la plus évidente consiste à éprouver actuellement de l'admiration pour Jeanne. C'est l'interprétation dispositionnelle de la phrase ci-dessus. Différemment, je peux vouloir dire qu'Eugénie éprouve à ce moment-là, de manière occurrente, de l'admiration pour Jeanne. C'est l'interprétation actuelle ou non dispositionnelle de la phrase en question.

Comme les intuitions sont des états intentionnels occurrents, les états affectifs qui leur ressemblent le plus sont clairement les émotions. S'il est des phénomènes affectifs dont les intuitions peuvent être rapprochées, ce sont bel et bien les émotions. Les humeurs et les dispositions affectives ne sont pas des candidats plausibles.

\subsection{La conception affective des intuitions à l'épreuve des desiderata}

La prochaine étape consiste évidemment à examiner si les émotions sont susceptibles de satisfaire les trois desiderata mentionnés ci-dessus. Commençons par la spécificité phénoménologique des intuitions qui, selon certains philosophes - comme nous l'avons indiqué - les distingue des croyances.

Quand les philosophes des émotions discutent de la phénoménologie de celles-ci, ils insistent le plus souvent sur le fait qu'éprouver une émotion c'est ressentir un certain nombre de troubles ou d'agitations corporels ou physiques. Si vous demandez à quelqu'un ce qui lui semble être le cas au moment où il est saisi par la peur, il vous répondra en premier lieu qu'il lui semble que son rythme cardiaque s'est accéléré, que ses mains sont devenues moites, etc. Cette phénoménologie corporelle des émotions ne semble pas caractériser les intuitions. Je reviens sur ce point plus bas $(\mathbb{S} 4.3)$. Il est évident que les sensations corporelles constituent une grande partie de la phénoménologie de certaines émotions, et qu'elles attirent la plus grande partie de notre attention, d'autant plus qu'elles peuvent être très intenses. Mais ces sensations corporelles épuisent-elles la phénoménologie des émotions? Est-ce que tout ce qui me semble être le cas lorsque Séverine a peur que le chien errant s'approche consiste en l'impression de subir des troubles corporels? Quand Séverine a peur du chien errant, une autre chose lui semble être le cas. Il lui semble que le chien errant est dangereux ${ }^{18}$. Les émotions ont une phénoménologie évaluative au sens où il nous semble toujours, lorsque nous éprouvons une émotion, que l'objet sur lequel porte cette émotion est doté d'une propriété évaluative: le chien nous semble dangereux

18. La thèse qui reflète cette caractéristique phénoménale est celle selon laquelle les émotions nous présentent ou nous donnent accès à certaines propriétés évaluatives (le dangereux, le honteux, le regrettable, l'admirable, le dégoûtant, etc.) de la même manière que les expériences perceptuelles nous donnent accès à certaines propriétés naturelles (le vert sapin, le carré, le mou, le salé, etc.). Cette thèse est très répandue chez les philosophes et les psychologues des émotions. Voir Deonna et Teroni 20I2a, 20I2b; de Sousa I987; Mulligan I998; Tappolet 2000, 2016 . 
dans le cas de la peur du chien, l'action nous semble honteuse dans le cas de la honte à l'égard d'une action, l'oubli nous semble regrettable dans le cas du regret à l'égard d'un oubli ${ }^{19}$.

Revenons-en maintenant aux intuitions. Comme nous l'avons présenté ci-dessus, la phénoménologie des intuitions est, selon Chudnoff, spécifique. Elle diffère de celle des croyances, et même de celle qui caractérise certaines expériences perceptuelles (le cas du baromètre). Dans le cas d'une intuition, pense Chudnoff, il nous semble non seulement qu'une certaine proposition est vraie, mais il nous semble également être conscient de ce qui fait que cette proposition est vraie. Est-ce également ainsi que fonctionne la phénoménologie évaluative des émotions? Autrement dit, est-il correct d'affirmer que, dans le cas de la peur de Séverine, il semble à celle-ci non seulement que le chien est dangereux, mais également être consciente de ce qui rend vrai la dangerosité du chien, c'est-à-dire, par exemple, son air affamé, ses grognements, ses babines salivantes? Nous pensons que oui. Cela est quasi immédiatement induit par le fait que chaque émotion est fondée sur ce que les philosophes ont appelé «une base cognitive ${ }^{20}$. Contrairement aux expériences perceptuelles qui, lorsqu'elles sont véridiques, nous fournissent un accès immédiat aux propriétés naturelles, les émotions ne nous donnent pas directement accès aux propriétés évaluatives. Séverine ne peut pas avoir peur du chien errant si elle ne croit pas que ce chien a l'air affamé, qu'il grogne, qu'il salive, etc. Autrement dit, Séverine ne peut pas avoir peur du chien errant si elle ne croit pas que ce chien possède un certain nombre de propriétés naturelles qui font qu'il est dangereux. De la même manière, Fabio ne peut pas regretter avoir triché sans croire que cette action possède quelque propriété naturelle qui la rend regrettable, par exemple, que cette action a attristé son professeur. L'émotion de Séverine et celle de Fabio sont ainsi fondées sur des croyances qui constituent leurs bases cognitives. Chaque épisode de peur, honte, regret, etc., à propos d'un objet particulier doit être ainsi fondée sur la croyance que cet objet possède certaines propriétés naturelles qui le rendent dangereux, honteux, regrettable, etc. Or, si tel est le cas, la phénoménologie des émotions recoupe bien celle que Chudnoff attribue aux intuitions ${ }^{21}$. Lorsque Séverine a peur du chien errant, il lui semble non seulement que le chien est dangereux, mais il lui semble également, via la base cognitive sur laquelle cette émotion est fondée, être

19. Cette propriété évaluative est souvent baptisée "objet formel» des émotions. Lorsque Séverine a peur que le chien errant s'approche, la dangerosité du chien est l'objet formel de la peur de Séverine et le chien son objet particulier (qu'on baptise parfois ainsi pour le distinguer de son objet formel).

20. Voir Deonna et Teroni 20I2, Mulligan I998; Tappolet 2016.

21. La phénoménologie des émotions recoupe la phénoménologie des intuitions mais n'est pas identique à celle-ci, puisque la phénoménologie des émotions comprend un certain nombre d'impressions corporelles que la phénoménologie des intuitions ne comprend pas. Nous revenons sur ce point plus bas. 
consciente de ce qui rend vrai la dangerosité du chien errant (son air affamé, ses grognements, ses babines salivantes, etc.). Et cela est toujours le cas pour toutes les émotions. La base cognitive des émotions garantit à ces dernières le genre de phénoménologie complexe qui, selon Chudnoff, caractérise les intuitions.

Examinons maintenant le deuxième desiratum mentionné ci-dessus: la modularité. Les émotions sont-elles modulaires? Comme le reconnaissent souvent les philosophes des émotions, cette question requiert une réponse positive ${ }^{22}$. Il est, par exemple, parfaitement possible d'être dégoûté par un plat servi dans une assiette un peu sale tout en ne croyant pas que ce plat est dégoûtant. Il est aussi parfaitement possible d'être effrayé par une musaraigne tout en ne croyant pas que cette musaraigne est dangereuse. Les émotions fonctionnent apparemment comme les intuitions de ce point de vue.

Passons maintenant au troisième desiratum: la capacité rationnelle. Les émotions ont-elles une capacité rationnelle? Là aussi, il est assez facile de montrer que la réponse correcte à cette question est positive. Il est parfaitement approprié de poser la question «pourquoi/pour quelle raison?» dans le cas des émotions. "Pourquoi as-tu peur du chien errant? Pour quelle raison as-tu honte de tricher?», etc. Les émotions satisfont au critère du pourquoi ${ }^{23}$ et sont, dès lors, susceptibles d'être évaluées quant à leur ir/rationalité ${ }^{24}$. Certaines émotions sont rationnelles, d'autres non. Dans la mesure où les intuitions ont une capacité rationnelle alors que les expériences perceptuelles n'en ont pas, il est plus plausible de rapprocher les intuitions des émotions - comme le suggère la conception affective - que de les identifier à des expériences perceptuelles - comme le propose la conception perceptive. Autrement dit, le fait que les émotions satisfassent le critère du pourquoi suffit à faire la preuve d'un certain avantage de la conception affective sur la conception perceptive des intuitions. Faire la preuve de cela est notre objectif principal dans cette section. Nous aimerions néanmoins tenter d'en dire plus.

Non seulement les émotions et les intuitions ont toutes deux une capacité rationnelle, mais elles sont toutes deux rendues rationnelles de manière similaire. Voyons cela. Tout d'abord, qu'est-ce qui fait qu'une émotion est rationnelle ou irrationnelle? Selon Deonna et Teroni - et cela nous semble

22. Voir principalement sur ce point Tappolet 20I6, chap. I.

23. Voir Deonna et Teroni 20I2a, chap. VIII, 20I2b; Tappolet 20I6, 40-I.

24. Comme dans le cas des intuitions, il faut prendre garde à ne pas confondre cette affirmation avec la thèse distincte selon laquelle les émotions sont susceptibles de justifier des croyances et des jugements évaluatifs, c'est-à-dire des croyances ou des jugements qui portent sur l'exemplification par l'objet particulier de l'émotion d'une certaine propriété évaluative. L'idée est, par exemple, que la peur de Séverine à l'égard du chien errant justifie sa croyance que le chien errant est dangereux. Voir Deonna et Teroni 20I2a, 20I2b; Mulligan I998; Tappolet 2016 . 
être très plausible - , les émotions sont rationnelles ${ }^{25}$ "si et seulement si le sujet est conscient des propriétés qui constituent une instance de la valeur pertinente " (Deonna et Teroni, 20 2 2b, 55).

Formulée dans la terminologie que nous avons employée ci-dessus, une émotion est rationnelle si et seulement si l'individu a, via sa base cognitive, l'impression que l'objet particulier de son émotion possède des propriétés naturelles qui constituent la propriété évaluative pertinente. La peur de Séverine face au chien errant est rationnelle dans la mesure où Séverine a l'impression - via sa base cognitive - que le chien possède certaines propriétés naturelles (l'air affamé, les grognements, etc.) qui font effectivement de ce chien un chien dangereux. Si Séverine n'avait pas cette impression, ou si elle avait plutôt l'impression que le chien qui s'approche d'elle a l'air inoffensif, sa peur serait irrationnelle. Par contre, si nous comprenons bien la thèse défendue par Deonna et Teroni, il n'est pas nécessaire que le chien possède, en réalité, les propriétés naturelles qui le rendraient dangereux pour que la peur de Séverine soit rationnelle. L'important est seulement qu'elle ait l'impression (éventuellement erronée) que le chien possède ces propriétés naturelles, et que les propriétés naturelles dont elle a l'impression constituent réellement un danger.

Cette conception de la justification des émotions reflète parfaitement les réponses que nous donnons lorsque l'on nous demande pourquoi/pour quelle raison nous éprouvons telle ou telle émotion. Nous mentionnons la plupart du temps, dans ces réponses, les impressions que nous avons en ce qui concerne certaines propriétés naturelles pertinentes. Par exemple, à la question: «Pour quelle raison as-tu peur de ce chien?", Séverine répondra: «Parce que j'ai l'impression qu'il a l'air affamé et qu'il grogne».

Rappelons maintenant comment la grand-mère de Jules et Albert répondent lorsqu'on leur demande pour quelle raison ils ont, respectivement, l'intuition que I. le sujet du cas des fausses granges ne sait pas qu'il s'agit là d'une vraie grange, et que 2 . chaque diamètre d'un cercle constitue l'une de ses lignes de symétrie. La grand-mère de Jules et Albert invoque, tous les deux, des états imaginatifs. Ces états imaginatifs semblent, dès lors, être les raisons pour lesquelles ils ont l'une ou l'autre de ces intuitions. Avant de rapprocher cette affirmation de ce qui vient d'être dit à propos de la condition de rationalité des émotions, il est important de traiter de la difficulté mentionnée ci-dessus. Les états imaginatifs de la grand-mère de Jules et d'Albert ne sont-ils pas seulement les raisons explicatives de leurs intuitions, c'est-à-dire, des raisons qui expliquent causalement l'occurrence de ces intuitions mais ne les rationalisent pas? Nous ne le pensons pas. Et cela

25. Deonna et Teroni parlent de critère de "justification" des émotions. Mais ils entendent par «justification » la même chose que ce que nous entendons par "rationalité » dans cette contribution. Voir note I pour une explication de ce pourquoi nous préférons parler de rationalité plutôt que de justification. 
est visible dans le fait que si nous demandons, par exemple, à Albert: «Pourquoi as-tu l'intuition qu'il est vrai que chaque diamètre d'un cercle constitue l'une de ses lignes de symétrie " (plutôt que «Pourquoi as-tu l'intuition que chaque diamètre d'un cercle constitue l'une de ses lignes de symétrie?»), il répondra de la même manière. Si ces états d'imaginer n'étaient que les causes de son intuition, il serait contraint de répondre différemment. Si je vous demande pourquoi ou pour quelle raison votre mère croit à la réincarnation et que vous me répondez "que voulez-vous, c'est son éducation", vous faites alors mention d'une raison explicative. Si je souhaite en fait vous interroger sur les raisons qui rationalisent sa croyance, je reformulerai ma question ainsi: "Quelle raison a votre mère de penser qu'il est vrai que les êtres vivants se réincarnent?". Votre réponse ne mentionnera pas alors l'éducation de votre mère mais plutôt, par exemple, le fait qu'elle croit reconnaître les traits de caractère de son propre père dans votre labrador. Autrement dit, votre réponse mentionnera les raisons de votre mère de croire qu'il est vrai que son père s'est réincarné.

Nous avons dit que, selon une certaine conception plausible de la rationalité des émotions, l'émotion d'un individu $S$ est rationnelle si et seulement si $S$ a l'impression, via la base cognitive de son émotion, que l'objet particulier de cette émotion possède certaines propriétés naturelles pertinentes. Il nous semble que quelque chose d'assez similaire est vraie de la rationalité des intuitions. Nous venons de voir que, dans le cas des intuitions, les raisons des individus sont souvent des états imaginatifs ${ }^{26}$. Ces états imaginatifs donnent à voir ou présentent à l'individu un certain nombre de choses - par exemple, des lignes de symétrie et des cercles - et c'est sur la base de ces états imaginatifs que l'individu a l'intuition qu'une certaine proposition est vraie. Il semble que les états imaginatifs jouent, dans le cas des intuitions, un rôle proche de celui que joue la base cognitive dans le cas des émotions. Tout d'abord, les états imaginatifs nous donnent à voir un certain nombre de choses dont la présentation à l'esprit est nécessaire pour ressentir telle ou telle intuition de la même manière que la base cognitive des émotions donne à voir certaines propriétés naturelles des objets particuliers dont la présentation à l'esprit est nécessaire pour éprouver des émotions. Ensuite, de ces états imaginatifs dépend — comme le suggère le fait que les individus répondent à la question: «Pourquoi as-tu l'intuition que $p$ est vraie? ? en mentionnant des états imaginatifs - la rationalité des intuitions. La rationalité de l'intuition d'un individu dépend de ce qui a été donné à voir ou de ce qui a été présenté, à cet individu, le plus souvent grâce à ce qu'il a imaginé.

26. Nous ne supposons pas que les états imaginatifs sont les seules raisons des intuitions envisageables, mais ces derniers jouent certainement un rôle prépondérant. 


\subsection{Objections}

Nous aimerions maintenant considérer deux difficultés posées par l'hypothèse selon laquelle les intuitions sont des états affectifs similaires à des émotions.

Premièrement, le lecteur pourrait objecter que les émotions ont des contenus non conceptuels et les intuitions des contenus propositionnels, et que, dès lors, assimiler les secondes aux premières fait problème. Il y a deux manières de répondre à cela. Tout d'abord, il est possible de rappeler que la thèse selon laquelle le contenu des émotions est inévitablement non conceptuel ne fait pas l'unanimité chez les philosophes des émotions. Elle est principalement défendue par les auteurs qui identifient les émotions à des expériences perceptuelles, mais pas, forcément, par les auteurs qui considèrent que les émotions sont des états mentaux sui generis. Ensuite, même si, par souci de cohérence, nous avons intentionnellement toujours attribué des contenus propositionnels aux intuitions, rien de ce nous affirmons dans cette contribution ne repose sur la thèse selon laquelle le contenu des intuitions est toujours propositionnel.

Les émotions sont des états affectifs qui sont individués par un certain nombre de caractéristiques. Le second problème posé par l'hypothèse selon laquelle les intuitions sont des états affectifs similaires à des émotions est que les intuitions ne semblent pas caractérisées par certaines des propriétés que les émotions possèdent généralement. Plus précisément, les intuitions ne semblent pas avoir (i) de phénoménologie corporelle, (ii) de valence, et (iii) de pouvoir motivationnel. Nous avons déjà décrit brièvement la phénoménologie corporelle des émotions. Présentons encore rapidement les deux autres caractéristiques. Il semble que chaque type d'émotion soit toujours ou bien positif, ou bien négatif. Par exemple, la joie et l'espoir sont des émotions positives, alors que la colère et la peur sont des émotions négatives. C'est cet aspect des émotions que les philosophes et les psychologues des émotions appellent la valence. Quant au pouvoir motivationnel des émotions, il désigne - comme son nom l'indique - le fait que les émotions ne nous présentent pas seulement des choses comme étant dotées de telle ou telle propriété évaluative. Elles nous poussent également à accomplir des actions.

Nous aimerions répondre à cette deuxième objection en insistant sur le fait que les propriétés mentionnées ci-dessus ne sont pas essentielles aux émotions, au sens où un état affectif devrait nécessairement posséder chacune d'elles pour être une émotion. En effet, il paraitrait, par exemple, incongru de considérer que le regret n'est pas une émotion, et pourtant, il n'est pas évident que le regret ait une phénoménologie corporelle. Autre exemple: la surprise est l'une des émotions les plus reconnues, et pourtant, cette dernière n'est pas clairement dotée d'une valence soit positive soit négative, comme semble l'impliquer le fait qu'il y ait de bonnes et de mauvaises surprises ${ }^{27}$. Quant au

27. Voir Meylan 20I4 pour une brève discussion de la valence de la surprise et plusieurs indications bibliographiques. 
pouvoir motivationnel, il n'est, dans ce cas également, pas clair que toutes les émotions en soient pourvues. Quel serait, par exemple, le pouvoir motivationnel de l'espoir?

La catégorie des émotions n'est pas une catégorie d'états mentaux délimitée par une liste non négociable de conditions nécessaires. Il y a évidemment un seuil à partir duquel un état mental se distingue trop des émotions paradigmatiques (la peur, la colère, la honte, etc.) pour être considéré comme une émotion. Mais il n'est pas certain que nous ayons atteint ce seuil avec les intuitions. Il est évident, par contre, que les intuitions ne sont pas des émotions paradigmatiques. C'est pour éviter de donner l'impression contraire que la conception affective évoquée plus haut parle seulement de similarité avec les émotions, et non pas d'identité. L'hypothèse que nous avons essayé d'étayer ci-dessus consiste seulement à affirmer que les intuitions sont des états affectifs similaires à des émotions. Les intuitions présentent certaines caractéristiques - leur phénoménologie, leur modularité, leur capacité rationnelle — communes avec les états affectifs spécifiques que sont les émotions, qu'ils ne partagent pas avec d'autres types d'états mentaux. Apparemment, la conception affective des intuitions mérite qu'on s'y attarde.

\section{Bibliographie}

Bealer, G. "On the Possibility of Philosophical Knowledge", Philosophical Perspectives, I0, I996, I-34.

—. "Intuition and the Autonomy of Philosophy", Rethinking Intuition: The Psychology of Intuition and Its Role in Philosophical Inquiry, M.R. DePaul and W.M. Ramsey (eds.), Rowman \& Littlefield, I998.

Chudnoff, E. "What Intuitions are Like", Philosophy and Phenomenological Research, 82, 3, 2011, 625-654.

- Intuition, Oxford University Press, 2013.

Deonna J.A. and F. Teroni. The Emotions. A Philosophical Introduction, Routledge, $2012 \mathrm{a}$.

—_. "From Justified Emotions to Justified Evaluative Judgements", Dialogue, 5 I, 20I 2 b, 55-77.

de Sousa R. The Rationality of Emotion, MIT Press, I987.

Goldman A. "Discrimination and Perceptual Knowledge", Journal of Philosophy 73, I976, 77I-79I.

—. "What is Justified Belief?" Knowledge and Justification, G. Pappas (ed.), D. Reidel Publishing Company, I979, I-23.

Goldman A. and J. Pust. "Philosophical Theory and Intuitional Evidence", Rethinking Intuition: The Psychology of Intuition and Its Role in Philosophical Inquiry, M.R. DePaul and W.M. Ramsey (eds.), Rowman \& Littlefield, I998. Huemer. Ethical Intuitionism, Palgrave Macmillan, 2008.

Meylan A. "Epistemic Emotions: a Natural Kind? ”, Epistemic Emotions, Philosophical Inquiries, 2, I, 20I4, I73-I90.

Mulligan K. "From Appropriate Emotions to Values", The Monist, special issue: “Secondary Qualities Generalised”, P. Menzies (ed.), I998, I6I-I 88. 
Nussbaum M. Upheavals of Thought: The Intelligence of Emotions, Cambridge University Press, $200 \mathrm{I}$.

Roeser S. Moral Emotions and Intuitions, Palgrave Macmillan, 20 I I.

Solomon R. "Emotions and Choice", Explaining Emotions, A. Rorty (ed.), University of California Press, I980, 25I-28I.

Tappolet Ch. Émotions et valeurs, Paris, Presses Universitaires de France, 2000.

- Emotions, Values and Agency, Oxford, Oxford University Press, 2016.

Williamson, T. The Philosophy of Philosophy, New York, Routledge, 2007. 